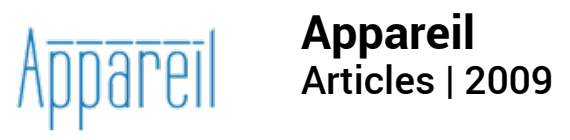

\title{
Le secret de « Septembre » Dans La Mort de Danton de Büchner
}

Littérature et événement à travers la pensée de Gilles Deleuze

\section{Ilai Rowner}

\section{(2) OpenEdition}

\section{Journals}

Édition électronique

URL : http://journals.openedition.org/appareil/876

DOI : 10.4000/appareil.876

ISSN : 2101-0714

Éditeur

MSH Paris Nord

Référence électronique

Ilai Rowner, «Le secret de "Septembre » Dans La Mort de Danton de Büchner », Appareil [En ligne], Articles, mis en ligne le 14 décembre 2009, consulté le 31 juillet 2020. URL : http://

journals.openedition.org/appareil/876 ; DOI : https://doi.org/10.4000/appareil.876

Ce document a été généré automatiquement le 31 juillet 2020.

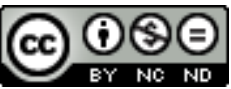

Appareil est mis à disposition selon les termes de la Licence Creative Commons Attribution - Pas d'Utilisation Commerciale - Pas de Modification 4.0 International. 


\title{
Le secret de « Septembre » Dans La Mort de Danton de Büchner
}

\author{
Littérature et événement à travers la pensée de Gilles Deleuze
}

Ilai Rowner

1 L'œuvre de Büchner, écrit Peter Szondi dans l'Essai sur le Tragique ${ }^{1}$, s'intitule La mort de Danton non seulement parce qu'elle présente les derniers jours de son héros, mais plus principalement parce que la mort constitue le problème propre du tragique. La mort n'est plus l'issue du destin tragique classique qu'on connaît chez Phèdre ou chez Hamlet, mais elle constitue le problème tragique lui-même. À savoir, écrit Szondi, « il n'y a aucune issue à une vie qui se vit comme état de mort ». Le paradoxe est construit dans cet ordre : la vie de Danton est devenue impossible à vivre, elle se retourne contre elle-même, mais puisque sa mort se confond avec sa vie, rien ne peut l'arracher à son destin. Danton ne cesse alors de mourir, ou bien il «ne peut mourir parce qu'il est déjà mort ${ }^{2} »$.

2 Danton est a priori destiné à la mort, l'opinion publique le condamne à la guillotine pour avoir mené une politique modérée et une vie bourgeoise. Certes il peut fuir, mais en se réfugiant, il sauvera sa propre mémoire, son « ennemi », celui qui le ronge, celui qui l'accuse des actes douteux du massacre des prisonniers royalistes et du clergé en septembre 1792, par lesquels il a acquis, paradoxalement, sa gloire révolutionnaire.

3 L'effondrement de Danton est à la fois privé et social, l'un suppose l'autre et l'un s'oppose à l'autre. Non seulement il éprouve une distance face à sa propre politique révolutionnaire, mais il souffre aussi d'une étrangeté plus profonde vis à vis de sa propre conscience personnelle. "Danton, nous dit Szondi, n'est pas seulement victime de la révolution en tant que révolutionnaire, il est victime aussi de lui-même en tant qu'homme. $»^{3}$

4 Szondi nous renvoie plus précisément aux deux scènes précédant l'arrestation de Danton. La quatrième scène du deuxième acte est un monologue en plein air dans lequel le conflit tragique est énoncé consciemment. On voit manifestement les hésitations qui l'empêchent de fuir ou de combattre, qui prévoient l'obsession de la mémoire ou qui aspirent à trouver le repos dans sa tombe. Cette formulation des 
arguments éclaire l'état actuel de Danton et guide éventuellement son action. En revanche - la cinquième scène est d'autant plus subtile - les éléments qui étaient explicitement analysés lors du monologue précédant surgissent involontairement pendant la nuit dans sa chambre tout comme un événement qui l'arrache hors de lui et qui l'expose à la violence de sa situation.

5 Szondi croit que la clé d'une interprétation psychologique et historique de la pièce toute entière réside dans cette scène. Là se révèle le plan caché de l'œuvre qui fournit l'explication à l'effondrement personnel et révolutionnaire de Danton. Il y est question non seulement de savoir pourquoi Danton se meurt tout en étant vivant, mais également par quoi et comment cela se produit, qui conditionne cet état. Jusqu'à ce point, Danton tenait un discours désillusionné qui laissait entendre une humeur mélancolique et fatiguée. Cette scène permet en revanche d'entrevoir quelque chose de la fêlure qui le tourmente, des forces immaitrisables qui le hantent. Dans l'intimité de sa chambre, dans les longues heures de l'insomnie, Danton est en proie à des hallucinations, la mémoire du massacre de « septembre » le poursuit sous la forme d'un cri impersonnel, la mémoire individuelle embrasse le présent collectif de la ville: "Quand je suis venu à la fenêtre - ça criait et braillait dans toutes les rues: Septembre! $»^{4}$.

6 Szondi nous signale par là que Danton est essentiellement sous l'emprise de la mort justement là où sa propre vie se détermine dans l'histoire. Non seulement ses devoirs historiques tournent en "péchés affreux", ce qui le dépouille de ses habits révolutionnaires, mais de ce fait, plus encore, il ne peut assumer ni comprendre sa propre «physionomie morale». Non seulement l'acte historique peut engendrer des remords et entraîner un regret, mais il comporte en lui, plus radicalement, le germe de la mort de celui qui le réalise. On comprend par là le paradoxe tragique de Danton, la présence irréductible de la mort qui trahit toute confiance et qui défait toute mesure de connaître l'expérience.

7 En d'autres termes, Szondi nous amène à concevoir dans l'apparition du "Septembre " une nécessité qui régit l'ensemble de la pièce. La révolution revendique pleinement son pouvoir par le massacre des royalistes et du clergé, et c'est également par cet acte qu'elle s'assigne sa propre fin. "Septembre » est le moment où Danton accomplit le mouvement de l'histoire, et c'est là tout autant que l'histoire le vainc. La nécessité tragique du "Septembre » suggère somme toute que l'histoire ne peut être vécue que par l'anéantissement de celui qui l'institue. En d'autres termes, «La Révolution est comme un Saturne, elle dévore ses propres enfants $»^{5}$.

8 C'est autour de cette idée que le drame personnel de Danton se déploie et que la confrontation politique et philosophique avec ses adversaires, Robespierre et Saint Juste, se concrétise. C'est donc par là que la mort de Danton devient inévitable et que Szondi considère finalement le cours des événements dans une visée obligatoirement fataliste. Danton lui-même en est conscient, sans pouvoir pour autant se soulager :

...Il faut que le scandale arrive, mais malheur à celui par qui le scandale arrive. Il faut, c'était ce même «il faut». Qui maudira la main sur laquelle est tombée la malédiction du «il faut»? Qui a prononcé «il faut », qui ? Qu'est-ce qui en nous fornique, ment, vole et tue ?

9 Cet « il faut » historique dont parle Danton, ce « il fallait du septembre 1792 », constitue donc la nécessité de l'événement de Danton. Nous venons de remarquer que Szondi l'évoque comme le plan caché qui préétablit la tragédie et qui assure sa cohérence par 
une conception de déterminisme paradoxal. Pour lui, la scène de "septembre " fait allusion à un contexte historique spécifique sur lequel se fonde le drame psychique de Danton et où se précisent les questions philosophiques et politiques de l'œuvre.

On se trouverait plus près d'une approche événementielle de la scène de "Septembre " par l'emprunt de la notion dramatique d'objective correlative que T.S. Eliot propose dans son court essai sur Hamlet ${ }^{7}$. Cette notion insiste sur l'importance de soutenir l'intensité émotive de l'héros tragique par un fait extérieur adéquat. Selon Eliot, l'expression des émotions devient immédiatement et parfaitement accessible à condition qu'elle s'accorde avec l'ensemble d'éléments qui mobilisent l'action: l'atmosphère, la situation, les objets sur lesquels le sentiment se projette. Pour que l'art acquière une nécessité, nous dit Eliot, il ne faut pas que l'émotion reste sans objet extérieur, donc trop abstraite, ni qu'elle excède son objet et devienne ainsi inexprimable. Par contre, l'émotion doit former une unité avec l'action extérieure: “The artistic 'inevitability' lies in this complete adequacy of the external to the emotion".

11 Là aussi, la scène de "Septembre " apporte une solution à l'état psychique de Danton. Si le mystère de la scène ne se résout pas ici par une explication historique, elle est dotée d'une intention animiste qui consiste à refléter la conscience du sujet dans l'univers qui l'entoure. Ainsi, les éléments objectifs se confondent avec l'expression des émotions de Danton: la nuit, le silence de la ville, le cri qui traverse les murs de la maison ou qui trouble sa pensée, le cri que Danton entend par la fenêtre ou qu'il émet à travers ses lèvres. Cette atmosphère soutient la mémoire sanglante de "Septembre »; le problème psychique du personnage baigne toute la scène, et forme par là une structure nécessaire qui justifie la souffrance de Danton et qui assure la cohérence de l'œuvre.

12 Comme Szondi, Eliot nous permet de considérer l'apparition de «Septembre » comme un événement indispensable qui condense la nécessité de l'œuvre. Szondi le situe dans la visée déterministe de l'ensemble de la pièce, alors que d'après la notion d'Eliot nous le comprenons plutôt comme une structure esthétique fondamentale dans laquelle l'adéquation entre l'émotif et l'objectif détermine la fiabilité de l'action. Cependant, Szondi et Eliot conçoivent tous les deux la question de la nécessité comme une unité cohérente qui impose un ordre au désordre créatif, ou comme un programme initial qui modalise les diverses circonstances dans une seule dynamique. Eliot et Szondi suggèrent l'illusion d'une plénitude créative qui a dû obligatoirement prendre une telle forme d'existence. L'œuvre doit être ce qu'elle est, un travail de rigueur et d'exactitude. L'événement doit être toujours ainsi, de sorte que les éléments contingents qui y participent ne servent que pour l'accomplir ou pour le confirmer.

13 Certes, l'œuvre doit acquérir une dimension nécessaire qui est le moteur du processus créatif et qui peut lui assigner une essence. Or, dans ce cas, chez Eliot comme chez Szondi, elle dépend d'une stratégie des lectures préalables, d'un plan caché ou d'une structure profonde qui domine la liberté créative et qui l'enferme dans une forme fixe. Le déroulement de la pièce suit un programme général, il accomplit un ensemble de conditions et d'actions dans le but d'atteindre un résultat spécifique. De sorte que les éléments contingents qui s'y trouvent ne réduisent pas la cohérence de l'œuvre mais la renforcent et l'enrichissent. En d'autres termes, tout ce qui reste inachevé, tout ce qui est imprévu, tout ce qui échappe à la règle dans la littérature, sera sous-estimé ou bien assimilé par une logique constante. 
14 La question de la nécessité d'une œuvre se pose différemment chez Gilles Deleuze. Deleuze n'exclurait pas probablement tout horizon d'attente qui détermine la lecture ou la création, mais il insisterait plutôt sur le fait que la nécessité d'une œuvre doit être éprouvée sans que soit niée sa propre construction contingente. Il s'agirait simplement de renverser la perspective : au lieu de chercher comment la contingence confirme la nécessité, il faudra restituer la valeur nécessaire de l'œuvre à partir de sa construction contingente. Il serait question de concevoir une nécessité à mesure que l'œuvre s'efforce de déborder ses propres conditions initiales, en soulevant non pas ce qui reste identique ou semblable, mais au contraire, les éléments étranges et les moments de différences qui exposent les failles de l'œuvre et l'emporte à ses extrémités, au bord même de la possibilité de son effondrement.

15 Avant de juger l'œuvre comme un objet achevé, cette approche nous invite à parcourir l'expérience artistique dans son inaccomplissement. Avant de l'analyser rétrospectivement comme un fait fini, la critique a besoin d'entrer dans l'œuvre comme à l'intérieur d'un corps à l'état vivant ${ }^{9}$, dans la continuité et dans la fragilité d'une expérience vivante. C'est en ce sens que nous empruntons à Deleuze le terme d'événement ${ }^{10}$. Avant même de se demander pourquoi l'œuvre est ce qu'elle est, l'événement envisage la littérature comme une "aventure » ouverte. Au lieu de voir dans l'œuvre une créature préformée et passive, il nous introduit au milieu de ce qui advient, afin d'admettre toute la créativité et l'agitation des accidents et des variations qui s'y jouent, afin de constater comment les éléments imprévus modifient le dessin de l'œuvre.

16 C'est pourquoi la scène de «Septembre " nous importe plus particulièrement comme point de départ d'une critique événementielle. Nous y assistons à une rupture dans le déroulement causal de la pièce et nous y sommes immergés par une force nouvelle et inconditionnée qui trouble les limites de ce qui s'est constitué jusqu'alors ${ }^{11}$. Les circonstances inattendues et inexplicables de cet événement nous donnent l'impression que ce qui se produit ne repose que sur le hasard, c'est-à-dire, sur ce qui a pu ne pas se produire du tout. L'événement s'ouvre et résiste tout autant à l'expérience de celui qui l'éprouve ${ }^{12}$, il appelle un mouvement d'appropriation tout en restant inapproprié par principe, tout en brouillant la possibilité de le mesurer. Car «ce qui fait événement procède d'une dissociation des effets et des causes $»^{13}$. Car pour la première fois les actions de Danton s'exposent sans intention, sans choix ni règle. Danton est pris dans le processus impersonnel de l'événement qui l'envahit et qui le détermine hors de luimême. L'événement ne dissout pas l'univers objectif de l'œuvre sans dissoudre également l'unité subjective du personnage principal.

Or, à mesure que l'événement survient dans toute sa puissance contingente, il nous impose de lui assigner une nécessité. Même s'il reste inintelligible, l'événement nous demande de capter les sensations et les forces qui apparaissent en lui. L'événement nous invite à les rassembler dans toute leur autonomie constructive, dans toute la condensation de leur rapport, afin d'éprouver la nécessité d'un mouvement vital, tel que Deleuze le définit dans le concept de devenir. La nécessité vitale d'un événement devrait donc dépendre d'une déviation incertaine dans l'organisation de la pièce, au cours de laquelle se dégageraient des nouvelles compositions de sensations, des nouveaux modes d'individuation qui seraient éventuellement capables de marquer la conscience constitutive de l'œuvre toute entière.

18 À ce stade, il faut proposer une nouvelle réflexion sur la scène de « Septembre ». 
Une chambre. C'est la nuit. Danton, à la fenêtre: Cela ne cessera donc jamais ? Cette lumière ne va-t-elle jamais s'éteindre et cet écho ne jamais pourrir, n'y aura-t-il jamais de silence et d'obscurité, que nous ne voyions plus et n'entendions plus les uns les autres nos répugnants péchés? - Septembre !14 Dans ces conditions, il nous faudra avoir recours à une modalité d'événement spécifique, l'Heccéité, qui permettra de saisir de plus près les forces qui sont appliquées dans la scène. Deleuze et Guattari réservent le terme d'heccéité ${ }^{15}$ pour désigner un mode d'individuation fait de coordonnées spatio-temporelles qui ne sont ni sujet ni chose.

20Avec ce terme, les scolastiques médiévaux et notamment Duns Scot posent le problème d'un être individué réfléchissant à ce qui, dans l'expérience, le rend particulier et à ce qui le distingue de tout autre individu. L'heccéité permet de penser à titre d'exemple comment l'espèce commune «Homme» se divise en propriétés autonomes et indivisibles de la personne " Jean ", «Pierre ", « Jérôme »"

21Chez Deleuze et Guattari le recours à l'heccéité est une occasion de concevoir une forme d'individualité qui ne soit ni une personne psychologique ni un sujet du langage. Si l'heccéité ne désigne pas la forme essentielle d'une personne, d'un sujet ou d'une chose, elle permet à Deleuze et Guattari de différencier la présence d'un événement accidentel qui traverse les sujets et les choses tout en les regroupant. En termes concrets, l'heccéité serait l'individualité d'un jour, d'une saison, d'une heure, d'une atmosphère, d'un air ; " un degré de chaleur, une intensité de blanc sont des parfaites individualités " écrivent-t-ils ${ }^{17}$. Et c'est à ce titre même que nous avons accès à l'heccéité de «Septembre » dans La Mort de Danton.

L'individualité d'un jour, d'une saison, d'un degré de chaleur ne serait donc ni un simple décor, l'arrière plan dans lequel le sujet se situe, ni l'objet sur lequel l'émotion se projette pour s'exprimer comme nous venons de voir avec Eliot. L'heccéité indique premièrement une coordonnée spatio-temporelle qui devient une entité parfaitement particulière: les peintres y sont bien sensibles. Delacroix voit les mouvements des couleurs qui imprègnent le lion avec le cheval et le chasseur. Cézanne cherche la sensation de la couleur du violet, la force de pesanteur de la montagne. Le peintre français Marchand dit dans un entretien: "Dans une forêt, j'ai senti à plusieurs

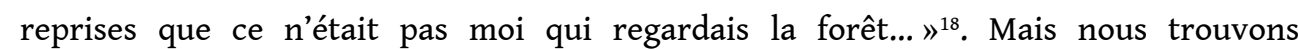
également des heccéités dans toute grande œuvre écrite, dans la présence du Minuit d' Igitur chez Mallarmé et dans les heures alphabétiques de Valéry : " Au commencement sera le sommeil. Animal profondément endormi; tiède et tranquille masse mystérieusement isolée $»^{19}$. On la trouve partout chez Proust. Ou alors, plus près de nous, chez Robbe-Grillet et le travail du regard dans La Jalousie. Et quand Claude Simon voit dans la lumière qui pénètre par la fenêtre la lettre $T$ l'initiale du mot temps se dessinant dans la chambre d'une vieille dame mourante.

Nous apercevons cependant que dans chacun de ces moments l'heccéité ne se sépare pas complètement du principe d'individuation du sujet ou de la chose. L'heccéité et le sujet s'entrelacent et cependant ils ne se fusionnent pas. Le sujet en fait partie et l'atmosphère se condense comme un tempérament. Il ne reste du sujet qu'un regard, des regards qui s'efforcent de voir, des yeux qui s'accoutument au noir, transperçant comme transpercés par l'univers, submergés, ensevelis. "J'apprends à voir, je ne sais 
pas pourquoi tout pénètre en moi plus profondément $»^{20}$ écrit Rilke. Et Yaïr Horowitz, un poète israélien : «Silencieux passe le soir / dans le cœur et dans l'arbre. $»^{21}$

L'heccéité est donc non seulement une coordonnée spatio-temporelle particulière, mais aussi une composition intensive des corps et des vies, un assemblage individué dans lequel le sujet devient un événement et l'atmosphère devient un corps, se donnant comme une entité de sensation.

Le climat, le vent, la saison, l'heure ne sont pas d'une autre nature que les choses, les bêtes ou les personnes qui les peuplent, les suivent, y dorment ou s'y réveillent. Et c'est d'une seule traite qu'il faut lire : la bête-chasse-à-cinq-heures. Devenir-soir, devenir-nuit d'un animal, noces de sang. Cinq heures est cette bête! Cette bête est cet endroit ! «Le chien maigre court dans la rue, ce chien maigre est la rue », crie Virginia Woolf. Il faut sentir ainsi. Les relations, les déterminations spatiotemporelles ne sont pas des prédicats de la chose, mais des dimensions de multiplicités ${ }^{22}$.

Il faut encore insister sur ce point : le mode d'individuation de l'heccéité repose sur les principes de composition et de multiplicité. Si un sujet ou une chose s'articulent dans l'individualité d'une heure ou d'un endroit, ils ne s'y positionnent pas simplement comme la cause première, psychologique ou sensible, ni comme le centre d'une composition linéaire, ni comme le sommet d'une composition pyramidale. Si en effet le sujet participe dans l'heccéité c'est que sa forme n'est plus celle d'un déjà advenu, d'un déjà constitué, mais qu'elle se laisse dissoudre et se recomposer dans les différentes relations à venir, dans la multiplicité advenante qui a lieu tant que le sujet est pris dans l'événement ${ }^{23}$.

Dans ces conditions, l'apprentissage d'une heccéité consiste à suivre une démarche descriptive de l'événement qui procéderait par l'entassement plutôt que par l'organisation des composantes, tout en mettant entre parenthèses le savoir qui se dégage de l'expérience, afin de remarquer au plus près quel est son mouvement singulier, et afin d'entrevoir, sans porter de jugement, sans hiérarchie et sans profondeur préalables, les divers objets et les rapports qui agissent dans la composition.

C'est dans cette perspective que nous entendons étudier la scène de "Septembre ". Nous la retrouvons en y lisant le lieu même d'une heccéité, qui est, plus particulièrement, celui d'une composition particulière entre le corps atmosphérique du «septembre» et le corps de Danton. La présence de "Septembre» envahit l'appartement de Danton, elle retentit à travers les murs, elle est partout dans la ville, et si elle témoigne de l'âme de Danton, elle se dit d'autant plus elle-même. "Septembre "c'est le moment où l'événement arrive à l'homme, le moment où l'homme parvient à se naître dans l'événement et de là provient également sa vitalité. «Septembre " nous semble être un cas concret d'heccéité, il nous montre comment l'heccéité se déploie, comment examiner sa composition, quelle est sa propre temporalité et comment son mouvement se dédouble entre le contingent et le nécessaire.

« Septembre » apparaît dans la durée. Il est une expression, il est prononcé, il est le cri de Danton. Ou bien, ce n'est pas Danton, mais ce sont les murs qui ont émis sa pensée, ce sont les « lèvres de pierres " qui ont parlé, les lèvres qui sont devenues froides. Or à y regarder de plus près il semble que «Septembre » vient de plus loin, d'auparavant, comme un écho qui ne cesse jamais, Danton s'est approché de la fenêtre et "ça criait ", 
du dehors, dans toutes les rues, un cri neutre, «n'y a-t-il pas eu un cri : septembre? », demande-t-il à Julie. «Oui, je l'ai entendu à travers toute la maison », répond-elle.

Désormais «Septembre " est un mot, c'est le contenu d'un mot, un mot qui regarde Danton d'un œil incompréhensible. Et puis, c'est un mot qui fait violence, qui faitsigne : "Pourquoi celui-là ?", ce signal-là ? Il tend ses mains trempées de sang, et Danton doit réagir d'emblée et se défendre : "je ne l'ai pas tué ». Il évoque un passé, il s'est passé quelque chose en septembre, "n'était-ce pas en septembre? ", que s'est-il passé en septembre? Il ne reste que le nom du mois, l'année est effacée et, quelques bribes de phrases: les rois, les aristocrates, la République, perdue, sauvée, nous les avons tués, il le fallait.

Enfin, « septembre » est cet «il faut » historique ou éthique, « c'était ce même il faut ", d'après lequel nous ne sommes plus nous-mêmes, en dessous duquel on devient quique-ce-soit, un quelqu'un ou un quiconque, « une marionnette » qui tient une épée et qui tente de trouver une identité dans l'anonymat: Qui est-ce? Qui l'a dit? Qu'est-ce qui est en nous? Une marionnette tirée par des forces inconnues, qui n'agit qu'en rapport à l'unique loi de pesanteur du «il faut », et pourtant elle le sait, "malheur à celui par qui le scandale arrive", par qui l'«il faut " arrive. Ce ne sont plus les circonstances mêmes du scandale qui importent, mais l'impossibilité d'y échapper, l'impossibilité de désobéir ou de consentir à ses lois, l'impossibilité qui multiplie les visages : Qui a fait? Qui a jugé ? Qui a condamné ?

Cependant, cette description de l'événement n'est pas tout à fait suffisante. L'observation des objets qui réagissent sous le signe de "Septembre" suppose un travail complémentaire qui consiste à déterminer leur relation, à localiser le mouvement qui les lie. Il est nécessaire de se demander, plus encore, quel est l'élan interne à l'événement ? Par quel motif l'entassement des éléments peut-il se produire? Est-ce le lien temporel des conséquences historiques? Est-ce le lien spatial d'un inventaire des phénomènes qui se placent visibles dans le présent ? Peut-on dire que la description est régie par le lien moral d'un péché originel ou d'une arrogance immortelle (hybris), qui conduirait Danton à révéler les ombres de ses actions et à reconnaître sa culpabilité ? Ou bien, est-ce un principe psychologique, des éléments refoulés, des souffrances personnelles, des déplaisirs qui conditionnent la conscience, qui reviennent à se manifester comme des pulsions inconnues qui menacent le soi ?

Tout ce que nous pouvons constater pour le moment c'est que «Septembre » advient comme une répétition. Qu'il se répète comme des reflets qui font superposer l'extérieur et l'intérieur, le mauvais rêve et l'état d'éveil, la victime et le bourreau. "Septembre » apparaît comme une révélation incertaine à la fois réelle et imaginaire, sur la fenêtre qui sépare la ville et la chambre, il retentit à travers les murs de la maison, d'un corps à un autre, de Danton à Julie.

«Septembre » n'est pas un fait historique ou un plan caché qui sous-tend le visible. Le massacre de Septembre 1792 n'apporte rien de spécifique, sauf en introduisant sur la scène la rumeur d'une violence déjà passée qui est encore à venir. "Septembre » est avant tout un cri, un mot, une mémoire non-déclarative, un secret qui se propage dans une durée répétitive. Tant que sa face antérieure se dévoile, tant que le passé se précipite à se découvrir, l'événement tarde à se donner tout entier et sa nécessité reste inconnue. Danton continuera à l'éprouver comme un «jamais vu» qui est toujours déjà-là, puisque «cequi répète, écrit Deleuze ailleurs, ne le fait qu'à force de ne pas "comprendre", de ne pas se souvenir, de ne pas savoir ou de n'avoir pas conscience. $»^{24}$ 
Ce double mouvement entre le «jamais vu » et le « déjà vu » illustre la définition que Deleuze confère à la temporalité de l'événement ${ }^{25}$. Le temps de l'événement est envisagé en tant que pur passage entre le passé et le futur, il est marqué par la division continuelle entre ce qui est déjà-passé et ce qui est encore à venir. Étant donné que l'instant de l'événement est saisi par son milieu, dans sa durée interne et dans l'intensité de sa croissance, il n'est jamais un instant discontinu, mais il se dédouble à la fois comme étant toujours déjà commencé sans jamais atteindre son achèvement. Il oscille entre ce que nous ne sommes pas encore et ce que nous cessons d'être.

De même ici, lorsque Danton est pris dans ce double piège de l'événement, dans cet espace creux, dans ce temps sans présent qui n'a ni commencement ni fin, quand il est capturé entre l'abîme de « qu'est-ce qui s'est passé ? » et l'incertitude de « qu'est-ce qui va se passer?». Danton ne peut se défaire de la sensation que quelque chose est préalablement arrivé, or ce n'est pas le passé en tant que tel qui le trouble, c'est le fait même que le passé continue, que le passé ne finit pas de se passer, qu'il se reproduit ou se déplace, qu'il devient différent, tel un cri, tel un signal, tel un secret, qui surgira bientôt, qui renversera bientôt tout ce qui vient.

Une fois de plus on pourra adhérer avec conviction au déterminisme de Szondi en disant que Danton n'a pas encore fini de faire la révolution alors que trop tôt la révolution le prend pour sa victime. Certes, peut-être Büchner lui-même n'a-t-il songé qu'à explorer la force de cette formule, mais il nous semble que dans ces conditions l'événement de "Septembre » se fixerait dans un processus fini, dont l'état initial et l'état terminal lui assigneraient une justification plus que son mouvement lui-même.

L'événement n'est pas un processus fini entre un passé et un avenir, mais le mouvement infini par lequel l'instant se divise, devance et retarde, par la double attraction du passé et du futur. Ainsi, plutôt qu'une information finie qui enrichit le programme de la pièce, «septembre » apparaît comme un secret perceptible. Tant qu'il se manifeste, la stupeur masque le passé et sa face antérieure disparaît. Tant que le passé se précipite à se répéter et à se découvrir, l'événement se suspend et se différencie, l'attente se prolonge.

«Septembre » est enfin l'instant où un corps étranger vient s'installer dans la pièce. Le nom d'un mois, d'une conscience atmosphérique indéfinie cherche à délivrer son énergie. C'est l'instant où la composition impersonnelle de l'heccéité se déploie, l'univers parle et l'imperceptible surgit visiblement. "Septembre» apparait ainsi comme un secret perceptible. Il ne s'agit pas simplement de remarquer que Danton a un secret qu'il faut révéler tout comme on ouvre une boîte de Pandore qui permettrait d'expliquer l'inconscient de l'œuvre et son fatalisme tragique. Il s'agit plutôt de laisser entrevoir un secret absolu. L'heccéité ne suggère pas des clés pour déchiffrer le contenu fini du secret de Danton, elle se manifeste, au contraire, comme une composition particulière qui fait voir « la forme infinie » du secret ${ }^{26}$.

L'heccéité investit le perçu, le contingent, pour déceler la nécessité de l'imperceptible. Le secret de "Septembre » est un inconnu qui transparait dans l'œuvre, qui trouble la matière de l'écriture tout en persistant en tant que tel. Sous la forme d'un secret absolu, l'événement devient donc une nécessité qui se construit accidentellement. À mesure que le secret résiste à son déchiffrement, les éléments contingents se multiplient tout en réfléchissant le poids d'une nécessité inouïe. À travers le secret, l'œuvre s'ouvre à la pression de ce qui l'excède tout en étant troublée par une nécessité qui n'apporte pas un soulagement. Certes, la stupeur menace l'établi, le désordre 
pénètre dans la création et cependant le nécessaire s'impose sans pour autant se nommer clairement. En quelque sorte, le secret expose l'impossible ou au moins l'improbable, ce qui pourrait arriver autrement se confond avec ce qui arrive éventuellement, et pourtant la nécessité trace une limite dans le chaos du hasard en y posant des exigences finies.

Il n'existe pas de rapport hiérarchique entre le nécessaire et le contingent qui ferait voir dans le nécessaire une pure essence ou bien un plan unique sous les manifestations contingentes. Le nécessaire du secret dépend simultanément de ses aspects contingents, l'imperceptible n'a d'autre existence qu'en devenant visible, sa densité est liée directement à sa dissémination. Le secret devient plus impénétrable et d'autant plus pénible à supporter qu'il vibre partout, dans des objets et des phénomènes variables. Tout se passe comme si la diversité des phénomènes qui apparaissent sous le signe de "Septembre " évoquait la dissimilitude d'un double de Danton. C'est là que "Septembre » est une instance générative d'une nouvelle individuation, car c'est Danton lui-même qui n'arrive pas à se reconnaître dans sa propre ombre ou son propre fantôme : qui était-il ? Qui sera-t-il ? Secret absolu qui nous laisse de nouveau dans l'attente d'une nécessité, avec la construction bouleversante du hasard. C'est pourquoi l'événement du secret " ne peut être autrement qu'il n'est » tout en s'affichant comme une composition mouvante et hétérogène. C'est là peut-être le propre de l'art, propose Deleuze avec Guattari tout en assignant à l'événement du secret une définition générale, "Peut-être est ce le propre de l'art, passer par le fini pour retrouver, redonner l'infini $»{ }^{27}$

42 «Septembre » est le moment où Büchner, Danton et le lecteur se voient éblouis devant l'infini du secret, capturés par lui. Le secret résiste, mais il ne cache pas son énergie, son mouvement vital. L'œuvre saisit et émet ce qui la traverse, le bruissement et la blessure qui l'affectent. C'est sous l'empreinte de l'événement de "Septembre " qu'on arrive à un point à partir duquel il n'y a plus de retour : l'identité de Danton se dissout et la honte de son époque, l'« accord monotone » et le «même habit » de sa civilisation se révèlent.L'apparition de "Septembre » n'est pas le jugement de l'histoire ou l'accusation de la mémoire qui oblige Danton à se résigner à la mort tout en restant vivant.Il s'agit, au contraire,d'un excès de vie qui l'arrache à sa place propre, à l'ordre révolutionnaire, et qui le retire en dehors de ses mouvements corporels, en dehors de l'histoire. Mais Danton, tout en se détachant, tout en étant arraché à sa place, n'arrive pas à se libérer dans l'événement, il est de plus en plus inséré dans l'histoire, il vient en elle, il subit la dénonciation de la révolution trop tôt sur son propre corps, en découvrant la prise de ses chaînes, quandil se reconnaît être l'homme par qui le malheur de l'histoire arrive, quand «Septembre» l'expose, plus que le tribunal révolutionnaire, à l'épreuve de sa " physionomie morale ".

43 Avant même que le drame historique se prolonge par l'arrestation et la mise à mort de Danton, l'événement de «Septembre » marque toute la pièce par la force corporelle qu'il introduit, par la physionomie moralequ'il constitue, par un même mouvement vivant qui subit et qui s'arrache à la fois, par ce combat, cette épreuve, dans lequel Danton ne peut accepter totalement l'«il faut» du "Septembre» ni se dégager complètement de ses besoins historiques. 


\section{BIBLIOGRAPHIE}

Büchner G., CEuvres complètes inédits et lettres, « La Mort de Danton », Seuil, Paris, 1988.

Deleuze G., Logique du sens, Minuit « Critique », Paris, 1969, p. 174-178, 190-197.

Deleuze G., Parnet C., Dialogues, Champ Flammarion, Paris, 1977.

Deleuze G., Guattari F., Mille Plateaux, Capitalisme et Schizophrénie II, « Devenir-Intense, DevenirAnimal, Devenir-Imperceptibe », Minuit « Critique », Paris, 1980.

Eliot T. S., The Sacred Wood, "Hamlet and His Problems" Faber \& Faber, 1920, p. 81-87.

Szondi P., Essai sur le Tragique, Circé, Paris, 2003, p. 128-135.

\section{NOTES}

1. P. Szondi, Essai sur le Tragique, Circé, Paris, 2003, p. 128-135.

2. Ibid, p. 133-135.

3. Ibid, p. 130

4. G. Büchner, Acte II, scène 5, p. 132.

5. Ibid, Acte I, scène 5, p. 118.

6. Ibid, Acte II, scène 6, p. 133.

7. T. S. Eliot, The Sacred Wood, "Hamlet and His Problems" Faber \& Faber, 1920, p. 81-87.

8. Ibid, p. 86

9. Cf. P. Valéry, « Questions de Poésie », « Au sujet du "Cimetière Marin” » in Variété III, p. 37-55, p. 59-72.

10. Cf. G. Deleuze, Logique du Sens, p. 174-178 ; Deleuze, Parnet, Dialogues, p. 77-81; Deleuze, Guattari, Mille Plateaux, p. 111-112, ch. VIII et X ; Qu'est-ce que la Philosophie ? p. 92, p. 107-108.

11. On peut également évoquer ici un autre moment fascinant dans la pièce, qui excède la trame principale et qui semble être aussi étrange et aussi pertinent que la scène de "Septembre ». Il est question du monologue unique de Marion, personnage qui reste complètement anonyme et dont les mots et la mélancolie s'ouvrent comme le seuil d'un trajet non exploré, tout en contribuant à la pièce sa pulsion interne et son humeur: "Le soir, j'étais à la fenêtre, je suis très sensitive, comme liée à tout ce qui m'entoure par une unique sensation, je sombrais dans les vagues du crépuscule... » Acte I scène 5, p. 115.

12. J. Derrida in Le « concept » de 11Septembre, Galilée, 2003, p. 139.

13. J. Baudrillard, Power Inferno, Galilée, 2003, p. 22-23.

14. G. Büchner, Acte II, scène 5, p. 131.

15. Cf. Deleuze, Guattari, Mille Plateaux, 309-326 ; Deleuze, Parnet, Dialogues, p. 109-111.

16. Cf. Encyclopédie philosophique universelle, "Heccéité », "individuation ", les notions philosophiques, Puf, Paris, 1998.

17. Deleuze, Guattari, Mille Plateaux, p. 319.

18. G. Charbonnier, le monologue du peintre, éd. de la Villette, Paris, 2002, p. 110-111.

19. P. Valéry, CEuvres I, Bibliothèque de la pléiade, 1957, « $A B C », p .1725$.

20. Rainer Maria Rilke, les Cahiers de Malte Laurids Brigge, 1929, pour la traduction française : Maurice Betz, Point Seuil, Paris, 1966, p. 13.

21. En hébreu, Recueil de poésie israélienne, éd. Aked, 1969. Notre traduction.

22. Deleuze, Guattari, Mille Plateaux, p. 321. 
23. Cf. A. Sauvagnargues, De l'Animal à l'Art dans la philosophie de Deleuze, PUF, 2004, p. 131. On peut évoquer ici la thèse de $C$. Romano qui propose de voir dans le sujet un "advenant ", bien qu'il reste encore dans une logique subjective : «ce participe présent substantivé (l'advenant) s'efforce de nommer davantage un processus en instance qu'une réalité constituée : moins un "sujet » au sens classique que des modes diversifiés de subjectivation par et à travers lesquels un « je » peut advenir, répondre de ce qui lui arrive à partir de ces noyaux de sens qui sont pour lui les événements »L'Evénement et le Monde, Puf, 1997, p. 2.

24. Deleuze, Différence et Répétition, Puf, Paris 1968, p. 26.

25. Deleuze, Logique du Sens, p. 190-197.

26. Deleuze, Guattari, Mille Plateaux, p. 353-354. Nous trouvons le même problème chez Derrida quand il commente le sceau de la date dans la poésie de Paul Celan : «le poème ne dévoile un secret que pour confirmer qu'il y a là du secret, en retrait, à jamais soustrait à l'exhaustion herméneutique. Secret sans hermétisme, il reste, et la date, hétérogène à toute totalisation interprétative.» (J.Derrida, Schibboleth, Galilée, p. 50) et Heidegger, et Blanchot: «... le dédoublement à l'intérieur duquel un tel événement se retire comme pour préserver le vide de son secret ». (L'espace littéraire, Gallimard, 1955, p. 202).

27. Deleuze, Guattari, Qu'est ce que la Philosophie ?, Minuit, Paris, 1991, p. 186.

\section{RÉSUMÉS}

Une nouvelle lecture de La Mort de Danton de Büchner, en particulier d'un moment extraordinaire dans la pièce, permettrait d'y lire une conception de l'œuvre littéraire comme événement. La littérature est un lieu privilégié pour accueillir dans son expression des événements, à savoir une composition impersonnelle des sensations et des forces dont il faut analyser les rapports et l'animation. L'ontologie de l'événement de Gilles Deleuze et, plus précisément, sa tentative pour concevoir des nouvelles formes d'individuation, qui rompt avec l'unité substantielle du sujet dans la tradition métaphysique, nous offre la possibilité de pénétrer dans la présence de l'événement de Danton et de mettre en évidence les divers éléments qui le compose. En-deça du cadre formel de la structure narrative, de l'organisation de l'œuvre et de son ordre représentatif, le terme d'événement indique pour la littérature la nécessité de saisir les sensations et les forces corporelles qui agissent à l'intérieur de ce qui arrive, de tirer les sous-éléments qui s'articulent dans le mouvement d'écriture pour produire un fait social, un fait historique ou un fait personnel. Si le terme d'événement évoque l'intuition active que quelque chose arrive, a dû arriver ou va bientôt arriver dans la littérature, il ne faut pas se contenter de rapporter le récit ni d'induire son message, mais il est nécessaire de maintenir son attente et sa surprise, de soulever son éclat et son secret, d'accéder au mouvement et au bruissement de son espace vital.

Isoler l'événement et rester dans sa propre durée serait assigner à la critique littéraire un cadre de recherche non formel, un cadre dont les contours se déplacent sans cesse et resteront toujours à découvrir. $\mathrm{Vu}$ de l'intérieur de son processus, l'événement est formé des composantes contingentes, nouvelles et imprévisibles dont les liens causals ne sont pas évidents. Et pourtant, l'existence même de l'événement dans la littérature doit être nécessaire. L'œuvre littéraire doit forcément acquérir une mesure qui associe les diverses composantes dans un ensemble indivisible, dans un ensemble unique de rigueur et d'exactitude. Que l'œuvre soit nécessaire, cette condition lui accorde la valeur déterminée d'une création qui « ne peut être autrement qu'elle n'est ", telle est du moins la définition aristotélicienne de la nécessité (gr. Anankaion), le 
caractère obligatoire d'une création dont toutes les parties se tiennent et qui se dit en somme ne pouvoir être autre que telle.

Suivant la perspective événementielle de la pièce de Büchner, nous nous proposerons de poser les conditions par lesquelles il serait possible de concilier l'aspect nécessaire et l'aspect contingent d'une œuvre littéraire. Mieux encore, il nous faudra introduire les notions de «nécessaire» et de «contingent» dans un mouvement de dépendance réciproque afin de se demander comment on peut accorder à l'œuvre une nécessité sans dominer sa construction contingente? Ou bien, au contraire, comment il est envisageable d'accéder au déploiement complexe d'une œuvre sans qu'elle tombe dans un chaos absolu.

INDEX

Mots-clés : contingence, Danton, événement, Georg Büchner, Gilles Deleuze, hasard, Heccéité, littérature, Mort, nécessité, objective-corrélative, œuvre, Peter Zondi, secret, Septembre 1792 